\title{
Primary progressive freezing gait
}

INSERM

\section{Source}

INSERM. (1999). Orphanet: an online rare disease and orphan drug data base. Primary progressive freezing gait. ORPHA:75567

Primary progressive freezing gait is a rare, heterogeneous, progressively incapacitating neurodeg enerative disease characterized by freezing of gait (usually during the first 3 years), later associating postural instability, eventually resulting in a wheelchair-bound state. Other features may include mild bradykinesia, rigidity, postural tremor, hyperreflexia, speech disorder and dementia. The disease is unresponsive to dopaminergic treatments. 